\title{
Endothelial dysfunction: The contribution of diabetes mellitus to the risk factor burden in a high risk population
}

\author{
Muluemebet Ketete $^{1}$, Rabia Cherqaoui ${ }^{2}$, Abid R. Maqbool ${ }^{1}$, John Kwagyan ${ }^{1}$, Shichen Xu ${ }^{1}$, \\ Otelio S. Randall ${ }^{1}$ \\ ${ }^{1}$ Department of Medicine, Division of Cardiovascular Medicine, Howard University, Washington DC, USA \\ ${ }^{2}$ Department of Medicine, Division of Endocrinology and Metabolism, Howard University, Washington DC, USA \\ Email: cherqaoui rabi@yahoo.com
}

Received 21 March 2013; revised 2 May 2013; accepted 17 May 2013

Copyright (C) 2013 Muluemebet Ketete et al. This is an open access article distributed under the Creative Commons Attribution License, which permits unrestricted use, distribution, and reproduction in any medium, provided the original work is properly cited.

\begin{abstract}
Background: Cardiovascular diseases (CVD) are the leading cause of morbidity and mortality in Western societies and are rapidly becoming a worldwide health problem. African-Americans have increased morbidity and mortality rates from CVD. Our study aimed to assess the effects of the CVD risk factors burden alone versus with diabetes mellitus in a high risk CVD population. Methods: The two study groups consisted of thirty seven diabetics and thirty seven non-diabetic African-Americans aged $\geq \mathbf{5 5}$ years without clinical atherosclerosis having similar cardiovascular risk factors (age, hypertension, hypercholesterolemia, smoking, and body mass index) except for diabetes mellitus. Brachial artery flow-mediated dilation (FMD), Nitroglycerin-mediated dilatation (NMD) and carotid intima-media thickness (IMT) were recorded in all subjects. Results: Endothelial function as assessed by the brachial artery FMD was significantly impaired in the diabetic group compared to the non-diabetic group $(7.8 \pm 5$ vs $3.3 \pm 4 ; p=0.0001)$. There were no differences in neither Nitroglycerinmediated dilatation (NMD) nor carotid intima-media thickness (IMT) in the diabetic and non-diabetic groups. Conclusion: The contribution of diabetes to the development of endothelial dysfunction in subjects with clustering of CVD risk factors may be early as indicated by significant functional changes preceeding structural vascular changes..
\end{abstract}

Keywords: Diabetes; Endothelial Dysfunction; Cardiovascular Risk Factors

\section{INTRODUCTION}

Type 2 diabetes mellitus (DM) has reached epidemic levels with an adult world prevalence estimated to be at 366 million in 2011, and with an expected increase to 552 million by 2030 [1].

These alarming predictions indicate a growing economic burden among patients and society more broadly. Hypertension (HTN) and DM constitute a potent duet for the development of atherosclerotic and arteriosclerotic cardiovascular diseases. Approximately one half of patients with type 2 diabetes die prematurely of a cardiovascular cause related to accelerated atherosclerosis [2]. Consequently, there has been an increased in emphasis on understanding the underlying mechanisms orchestrating the onset of cardiovascular diseases (CVD) in diabetic individuals and identifying patients at a particular high risk for future cardiac events. Endothelial dysfunction constitutes an important early occurrence in the development of the atherosclerotic process as well as an independent predictor of poor prognosis in CVD $[3,4]$. The main aim of this study was to investigate the impact of DM on vascular structure and function as reflected by carotid intima-media thickness (IMT), brachial artery flow mediated dilatation (FMD) respectively. With this goal in mind, we studied 2 groups of patients without clinical atherosclerosis having similar cardiovascular risk factors (age, hypertension, hypercholesterolemia, smoking, and body mass index) except for diabetes mellitus.

The independent mechanistic contribution of diabetes mellitus to the risk factor burden for the development of arteriosclerosis and atherosclerotic lesions in a high-risk population that may result in impairment of arterial function has not been well studied.

\section{METHODS}

Two study groups were analyzed for this report: One group consisted of thirty seven diabetics and a second group of thirty seven non-diabetic African-Americans 
aged over 55 years without clinical CAD/CVD matched for age, gender, smoking, body mass index (BMI), low density lipoproteins (LDL) and triglycerides. The study protocol was approved by the institutional review board and each subject gave written informed consent.

\subsection{Flow-Mediated Dilation}

Flow-mediated dilation (FMD) of the brachial artery was measured as previously described [5]. Briefly, following measurement of the baseline diameter of the brachial artery, ischemic condition was induced by inflating a blood pressure tourniquet located at the proximal forearm to at least $50 \mathrm{mmHg}$ above the systolic blood pressure. After 5 minutes of pre-hyperemia ischemia, the tourniquet cuff pressure was released and 2D images of the brachial artery dilation following reactive hyperemia were recorded.

Endothelium-dependent dilatation (FMD) was defined as the percent change in arterial diameter following reactive hyperemia compared to the baseline diameter and calculated as follows:

$$
\begin{aligned}
& \% \mathrm{FMD}=100 \times \\
& \frac{(\text { diameter during reactive hyperemia }- \text { resting diameter })}{\text { resting diameter }}
\end{aligned}
$$

\subsection{Nitroglycerin-Mediated Dilatation (NMD)}

Nitrate-mediated dilatation (NMD) capacity (endothelium independent) dilation capacity was tested to examine the vasodilatory effect of an exogenous source of nitric oxide [5]. Changes in brachial artery diameter were measured in response to administration of $0.4 \mathrm{mg}$ of sublingual nitroglycerin, a direct smooth-muscle dilator. The endothelium-independent dilatation (NMD) was defined as the percent increase in arterial diameter 5 minutes following nitroglycerin compared to baseline and calculated as follows:

$$
\begin{aligned}
& \% \text { NMD } \\
& =100 \times \frac{(\text { maximum diameter }- \text { resting diameter })}{\text { resting diameter }} .
\end{aligned}
$$

\subsection{Carotid Intima-Media Thickness}

The carotid intima-media thickness (IMT) was evaluated using high resolution ultrasound. All patients were studied in a fasting state and all underwent a full clinical examination. Total cholesterol, triglycerides, and HDL cholesterol were determined by enzymatic methods, and LDL cholesterol was calculated using the Friedewald equation.

\section{RESULTS}

Data are expressed as means $\pm \mathrm{SD}$. Comparisons be- tween groups were analyzed by the unpaired Student t-test). P-values $\leq 0.05$ were accepted as statistically significant. Data processing was performed with the software modules of SPSS ${ }^{\circledR}$ (Statistical package for analysis in social sciences, Predictive analysis software release 18, SPSS Inc., Chicago, USA).

The clinical characteristics and biochemical profile of the study subjects are summarized in Table 1. There were no statistically significant differences between the groups in age, gender, smoking status, and BMI, however HDL was significantly higher in the non-diabetic group.

Endothelial dysfunction assessed by the brachial artery FMD was significantly impaired in diabetics compared to non-diabetics; $\mathrm{p}<0.01$ (Table 2).

The mean carotid IMT of diabetic subjects $(0.92 \pm$ $0.17 \mathrm{~mm}$ ) was similar to that of the RF-matched group $(0.94 \pm 0.16 \mathrm{~mm}$; no statistical difference). The NMD tended to decrease in diabetics; however, the decrease was not statistically significant.

\section{DISCUSSION}

Adult patients with diabetes are known to have higher rates of cardiovascular complications [6,7]. Type 2 diabetes mellitus (T2DM), hypertension (HTN), and obesity (OB) are all major risk factors for the development of cardiovascular-renal dysfunction (CVR-D). The greater

Table 1. Characteristics and metabolic profile of participants with and without DM.

\begin{tabular}{cccc}
\hline Variable & Non-diabetic $(\mathrm{n}=37)$ & Diabetes $(\mathrm{n}=37)$ & \\
\hline Age, Years & $65 \pm 6$ & $65 \pm 6$ & $\mathrm{~ns}$ \\
BMI kg/m & $31.7 \pm 6$ & $33 \pm 6$ & $\mathrm{~ns}$ \\
SBP, mmHg & $128.0 \pm 13$ & $130 \pm 14$ & $\mathrm{~ns}$ \\
DBP, $\mathrm{mmHg}$ & $77.0 \pm 8$ & $72 \pm 9$ & $<0.01$ \\
PP, $\mathrm{mmHg}$ & $51 \pm 4$ & $58 \pm 3$ & $<0.01$ \\
HDL, mg/dl & $52 \pm 11$ & $46 \pm 12$ & $<0.01$ \\
\hline
\end{tabular}

Table 2. Carotid IMT mm thickness and FMD\% changes in brachial artery diameter following ischemia \& nitroglycerin.

\begin{tabular}{lccc}
\hline \multicolumn{1}{c}{ Variables } & Non-diabetics $(\mathrm{n}=37)$ & Diabetics $(\mathrm{n}=37)$ & $\mathrm{p}$ \\
\hline $\begin{array}{l}\text { \% D Change } \\
\text { (FMD) }\end{array}$ & $7.80 \pm 5.00$ & $3.30 \pm 4.00$ & $<0.01$ \\
& $0.94 \pm 0.16$ & $0.92 \pm 0.17$ & $\mathrm{~ns}$ \\
IMT, mm & & & \\
$\begin{array}{l}\text { \% Nitroglycerin } \\
\text { Change }\end{array}$ & $9.90 \pm 6.00$ & $8.30 \pm 6.00$ & $\mathrm{~ns}$ \\
\hline
\end{tabular}


prevalence of CVR-D when a constellation of HTN, T2DM, and obesity are present is suggestive of synergistic effects of these risk factors on outcomes [8]. The higher incidence of vascular events in diabetics usually implies the presence of more frequent and severe cardiovascular risk factors.

Ample evidence exists that the clustering of an increasing number of cardiovascular risk factors in an individual has an impact on the morphological and functional changes of the vasculature [9-12], however, only a few studies have analyzed the differential impact of diabetes mellitus on the initiation and progression of endothelial dysfunction. Tsuchiya et al. [13] investigated 101 subjects with T2DM and reported that only FMD showed significant changes in diabetic subjects as risk factors accumulated as compared to other vascular parameters namely carotid IMT. They further demonstrated that insulin resistance showed the most significant association with reduced FMD. Previously published studies support the hypothesis that FMD may be one of the major determinants of development of CVD in both high and lowrisk populations and may provide additional prognostic value beyond traditional cardiovascular risk factors [1418]. On the other hand, endothelial-independent vasodilation with nitroglycerin administration was mildly reduced in diabetic subjects although this observation was not statistically significant. Such findings are consistent with the trend toward a reduced NMD response documented in earlier studies in diabetics [19-21] in which the impairment of endothelial function was much greater than that of the smooth muscle dilator response. Furthermore, while vascular smooth muscle cell dysfunction (NMD) has been previously well documented in diabetic patients compared to healthy controls [22,23], impairment of nitroglycerin responses has also been reported in adults with multiple CVD risk factors [24]. Hence, we investigated 2 groups of subjects, a diabetic and a nondiabetic group) sharing similar atherosclerotic risk factors: Age, gender, body mass index (BMI), smoking habit, hypertension, and lipid parameters. In the present study, endothelium-dependent brachial artery response to occlusion induced ischemia (FMD) was significantly diminished in diabetic patients when compared to high-risk individuals that have not manifested cardiovascular disease. These observations suggest that diabetes may be associated with reduced FMD/endothelial damage earlier than any of the traditional cardiovascular risk factors. In accordance with our findings, a recent study called attention to the fact that impaired endothelial function in diabetic patients was not related to the presence or levels of various established cardiovascular risk factors but rather to the duration of DM and found that for every 10 years of DM, FMD was reduced by $1 \%$ [25]. In a hypertension clinic, while evaluating random hypertensive patients for isolated systolic and diastolic HTN, the pulse pressure was noted to be larger in diabetics [26]. A wide pulse pressure (PP) is associated with CVR-D, and has been observed in DM patients; a comparative analysis of hypertension clinical trials including 62,712 diabetics and 108,599 non-diabetics revealed lower mean levels of diastolic blood pressure in diabetics and higher mean levels of systolic blood pressure [27].

This study has a few limitations: This study is small and multiple regression analysis was not done to determine the relative contribution of other risk factors.

\section{CONCLUSION}

The results of this study demonstrated that the contribution of diabetes to the development of endothelial dysfunction in subjects with clustering of metabolic factors is early, preceding structural changes, and contributes significantly to reduce FMD. Hence, FMD may be used as a sensitive marker for the early detection of atherosclerotic changes in diabetic patients with multiple cardiovascular risk factors.

\section{ACKNOWLEDGEMENTS}

This research was supported in part by NIH/NCRR grant number UL1RR031975 from the Design, Biostatistics \& Population Studies Component of the Georgetown-Howard Universities Center for Clinical \& Translational Science.

\section{REFERENCES}

[1] Roger, V.L., Go, A.S., Lloyd-Jones, D.M., Benjamin, E.J., Berry, J.D., Borden, W.B., et al. (2012) Heart disease and stroke statistics - 2012 update: A report from the American Heart Association. Circulation, 125, e2-e220. doi:10.1161/CIR.0b013e31823ac046

[2] Van Dieren, S., Beulens, J.W., van der Schouw, Y.T., Grobbee, D.E. and Neal, B. (2010) The global burden of diabetes and its complications: An emerging pandemic. European Journal of Cardiovascular Prevention \& Rehabilitation, 17, S3-S8.

doi:10.1097/01.hjr.0000368191.86614.5a

[3] Takase, B., Matsushima, Y., Uehata, A., Ishihara, M. and Kurita, A. (2008) Endothelial dysfunction, carotid artery plaque burden, and conventional exercise-induced myocardial ischemia as predictors of coronary artery disease prognosis. Cardiovascular Ultrasound, 6, 61. doi:10.1186/1476-7120-6-61

[4] Yeboah, J., Folsom, A.R., Burke, G.L., Johnson, C., Polak, J.F., Post, W. and Lima, J.A. (2009) Predictive value of brachial flow mediated dilation for incident cardiovascular events in a population-based study: The multiethnic study of atherosclerosis. Circulation, 120, 502-509. doi:10.1161/CIRCULATIONAHA.109.864801

[5] Kwagyan, J., Hussein, S., Xu, S., Ketete, M., Maqbool, A.R., Schneider, R.H. and Randall, O.S. (2009) The rela- 
tionship between flow-mediated dilatation of the brachial artery and intima-media thickness of the carotid artery to Framingham risk scores in older African Americans. The Journal of Clinical Hypertension, 11, 713-719. doi:10.1111/j.1751-7176.2009.00175.x

[6] Liu, J., Sempos, C., Donahue, R.P., Dorn, J., Trevisan, M. and Grundy, S.M. (2005) Joint distribution of non-HDL and LDL cholesterol and coronary heart disease risk prediction among individuals with and without diabetes. Diabetes Care, 28, 1916-1921. doi:10.2337/diacare.28.8.1916

[7] Fox, C.S., Coady, S., Sorlie, P.D., D’Agostino Sr., R.B., Pencina, M.J., Vasan, R.S., Meigs, J.B., Levy, D. and Savage, P.J. (2007) Increasing cardiovascular disease burden due to diabetes mellitus: The Framingham heart study. Circulation, 115, 1544-1550.

doi:10.1161/CIRCULATIONAHA.106.658948

[8] Lloyd-Jones, D.M., Leip, E.P., Larson, M.G., D’Agostino, R.B., Beiser, A., Wilson, P.W., Wolf, P.A. and Levy, D. (2006) Prediction of lifetime risk for cardiovascular disease by risk factor burden at 50 years of age. Circulation, 113, 791-798. doi:10.1161/CIRCULATIONAHA.105.548206

[9] Yokoyama, H., Sone, H., Saito, K., Yamada, D., Honjo, J. and Haneda, M. (2011) Flow-mediated dilation is associated with microalbuminuria independent of cardiovascular risk factors in type 2 diabetes-interrelations with arterial thickness and stiffness. Journal of Atherosclerosis and Thrombosis, 18, 744-752. doi:10.5551/jat.7526

[10] Wagenknecht, L.E., D'Agostino Jr., R., Savage, P.J., O'Leary, D.H., Saad, M.F. and Haffner, S.M. (1997) Duration of diabetes and carotid wall thickness. The Insulin Resistance Atherosclerosis Study (IRAS). Stroke, 28, 999-1005. doi:10.1161/01.STR.28.5.999

[11] Ishibashi, Y., Takahashi, N., Shimada, T., Sugamori, T., Sakane, T., Umeno, T., Hirano, Y., Oyake, N. and Murakami, Y. (2006) Short duration of reactive hyperemia in the forearm of subjects with multiple cardiovascular risk factors. Circulation Journal, 70, 115-123. doi:10.1253/circj.70.115

[12] Hashimoto, M., Kozaki, K., Eto, M., Akishita, M., Ako, J., Iijima, K., Kim, S., Toba, K., Yoshizumi, M. and Ouchi, Y. (2000) Association of coronary risk factors and endothelium-dependent flow-mediated dilatation of the brachial artery. Hypertension Research, 23, 233-238. doi:10.1291/hypres.23.233

[13] Tsuchiya, K., Nakayama, C., Iwashima, F., Sakai, H., Izumiyama, H., Doi, M. and Hirata, Y. (2007) Advanced endothelial dysfunction in diabetic patients with multiple risk factors; importance of insulin resistance. Journal of Atherosclerosis and Thrombosis, 14, 303-309. doi:10.5551/jat.E525

[14] Corrado, E., Rizzo, M., Coppola, G., Muratori, I., Carella, M. and Novo, S. (2008) Endothelial dysfunction and carotid lesions are strong predictors of clinical events in patients with early stages of atherosclerosis: A 24-month follow-up study. Coronary Artery Disease, 19, 139-144. doi:10.1097/MCA.0b013e3282f3fbde

[15] Yeboah, J., Crouse, J.R., Hsu, F.C., Burke, G.L. and Her- rington, D.M. (2007) Brachial flow-mediated dilation predicts incident cardiovascular events in older adults: The Cardiovascular Health Study. Circulation, 115, 23902397. doi:10.1161/CIRCULATIONAHA.106.678276

[16] Brevetti, G., Silvestro, A., Schiano, V. and Chiariello, M. (2003) Endothelial dysfunction and cardiovascular risk prediction in peripheral arterial disease: Additive value of flow-mediated dilation to ankle-brachial pressure index. Circulation, 108, 2093-2098. doi:10.1161/01.CIR.0000095273.92468.D9

[17] Chan, S.Y., Mancini, G.B., Kuramoto, L., Schulzer, M., Frohlich, J. and Ignaszewski, A. (2003) The prognostic importance of endothelial dysfunction and carotid atheroma burden in patients with coronary artery disease. Journal of the American College of Cardiology, 42, 10371043. doi:10.1016/S0735-1097(03)00927-6

[18] Shechter, M., Issachar, A., Marai, I., Koren-Morag, N., Freinark, D., Shahar Y., Shechter A. and Feinberg M.S. (2009) Long-term association of brachial artery flow-mediated vasodilation and cardiovascular events in middleaged subjects with no apparent heart disease. International Journal of Cardiology, 134, 52-58. doi:10.1016/j.ijcard.2008.01.021

[19] Woodman, R.J., Playford, D.A. and Watts, G.F. (2006) Basal production of nitric oxide (NO) and non-NO vasodilators in the forearm microcirculation in Type 2 diabetes: Associations with blood pressure and HDL cholesterol. Diabetes Research and Clinical Practice, 71, 59-67. doi:10.1016/j.diabres.2005.05.008

[20] Jawa, A., Nachimuthu, S., Pendergrass, M., Asnani, S. and Fonseca, V. (2006) Impaired vascular reactivity in African-American patients with type 2 diabetes mellitus and microalbuminuria or proteinuria despite angiotensinconverting enzyme inhibitor therapy. The Journal of Clinical Endocrinology \& Metabolism, 91, 31-35. doi: $10.1210 /$ jc. $2005-1632$

[21] Watts, G.F., O’Brien, S.F., Silvester, W. and Millar, J.A. (1996) Impaired endothelium-dependent and independent dilatation of forearm resistance arteries in men with diettreated non-insulin-dependent diabetes: Role of dyslipidaemia. Clinical Science, 91, 567-573.

[22] Lockhart, C.J., Agnew, C.E., McCann, A., Hamilton, P.K., Quinn, C.E., McCall, D.O., Plumb, R.D., McClenaghan, V.C., McGivern, R.C., Harbinson, M.T. and McVeigh, G.E. (2001) Impaired flow-mediated dilatation response in uncomplicated type 1 diabetes mellitus: Influence of shear stress and microvascular reactivity. Clinical Science, 121, 129-139. doi:10.1042/CS20100448

[23] Meyer, M.F., Lieps, D., Schatz, H. and Pfohl, M. (2008) Impaired flow-mediated vasodilation in type 2 diabetes: Lack of relation to microvascular dysfunction. Microvascular Research, 76, 61-65. doi:10.1016/j.mvr.2008.03.001

[24] Celermajer, D.S., Sorensen, K.E., Bull, C., Robinson, J. and Deanfield, J.E. (1994) Endothelium-dependent dilation in the systemic arteries of asymptomatic subjects relates to coronary risk factors and their interaction. Journal of the American College of Cardiology, 24, 14681474. doi:10.1016/0735-1097(94)90141-4 
[25] Naka, K.K., Papathanassiou, K., Bechlioulis, A., Kazakos, N., Pappas, K., Tigas, S., Makriyiannis, D., Tsatsoulis, A. and Michalis, L.K. (2012) Determinants of vascular function in patients with type 2 diabetes. Cardiovascular Diabetology, 11, 127. doi:10.1186/1475-2840-11-127

[26] Ferguson 3rd, J.J. and Randall, O.S. (1986) Systolic, diastolic, and combined hypertension. Differences between groups. Archives of Internal Medicine, 146, 1090-1093. doi:10.1001/archinte.1986.00360180072012

[27] Osher, E. and Stern, N. (2008) Diastolic pressure in type 2 diabetes: Can target systolic pressure be reached without "diastolic hypotension?" Diabetes Care, 31, 249-254. doi: $10.2337 / \mathrm{dc} 08$-s 262 